\title{
Newly Arising Contralateral Granular Cell Tumor after Wide Excision of Large Breast Granular Cell Tumor: A Case Report
}

\author{
Hyuk Mun Kim, Ok Pyeng Song, Jong Min Kim \\ Department of Surgery, Min General Surgery Hospital, Seoul, Korea
}

Granular cell tumor (GCT) of the breast is a rare neoplasm that is usually benign in nature. The results of physical examination and ultrasonographic findings of GCT of the breast often mimic those of other malignancies. A 46-year-old woman underwent wide excision for removal of a large mass in the left breast (about $10 \mathrm{~cm}$ in diameter). The pathologic finding of the excised mass was benign GCT. A year after initial surgery, recurrence of the left breast mass was found along with a newly developed mass in the contralateral right breast, which was excised. In this study, we describe the case of a newly developed GCT in the contralateral breast 1 year after excision of a larger breast GCT.

Key Words: Breast, Granular cell tumor, Recurrence

\section{INTRODUCTION}

Granular cell tumor (GCT) is a rare neoplasm that originates from Schwann cells in the nervous system. Although GCT can arise at any site in the body, it is usually found in the oral cavity, skin, or the digestive or respiratory tracts [1,2]. GCT predominantly occurs in women and may arise in the breast, and closely resemble carcinoma clinically, radiologically, and ultrasonographically $[3,4]$. The mammographic findings of GCT are those of an ill-defined heterogeneous dense mass [3], whereas ultrasonography reveals the presence of an irregularly shaped mass-like malignancy [4]. Some authors have previously reported GCT of the breast, but to the best of our knowledge, there is no report of a newly developed GCT in the contralateral breast after wide excision of a previous GCT lesion. Here, we describe a case of newly arising GCT in the contralateral breast 1 year after excision of a large breast GCT.

\section{CASE REPORT}

A 46-year-old woman presented with a painful, palpable large left

\section{Correspondence: Jong Min Kim}

Department of Surgery, Min General Surgery Hospital, 187 Dobong-ro, Gangbukgu, Seoul 01170, Korea

Tel: +82-2-1899-7529, Fax: +82-32-469-6319,

E-mail: goodbreastdoctor@gmail.com

Received: Nov 23, 2016 Revised: Feb 14, 2017 Accepted: Mar 6, 2017 breast mass that extended to the ipsilateral axilla. She had incidentally found a small lump in the upper lateral quadrant of her left breast 10 years prior to the presentation, but she did not seek medical advice because she did not experience any pain and the mass grew slowly. However, 1 month before this initial presentation, the patient felt blunt pain on the left breast lesion. On physical examination, the mass was found to be firm, well-defined, fixed, and about $10 \mathrm{~cm}$ in size with bulging of the overlying skin. Chest posterior-anterior radiography showed a large mass shadow, and ultrasonography revealed the presence of a large homogeneous solid mass in the left axillary area (Figure 1), but no mass or abnormal findings in the right breast. The patient had no history of surgery or use of any medication. Wide excision (Figure 2) was performed under general anesthesia, and pathologic findings revealed the mass to be a benign GCT (Figure 3), with tumor-free resection margins. Immunohistochemistry of the resected specimen was positive for Ki-67 (less than 10\% of tumor cells), S-100, and CD-68, but negative for cytokeratin and vimentin (not illustrated). No postoperative complication was observed, and the patient reported that he did not have any discomfort. Six months after the surgery, we found $1-\mathrm{cm}$ masses in both breasts and the patient was closely observed. A year after the initial surgery, the left breast mass grew to $3 \mathrm{~cm}$ in the previous operated site, and the contralateral well-defined mass, which was approximately $1.2 \mathrm{~cm}$ in size, was in the upper lateral quadrant of the right breast (Figure 4). Excision biopsy of both breast masses was performed and the histopathologic results were similar to those observed 

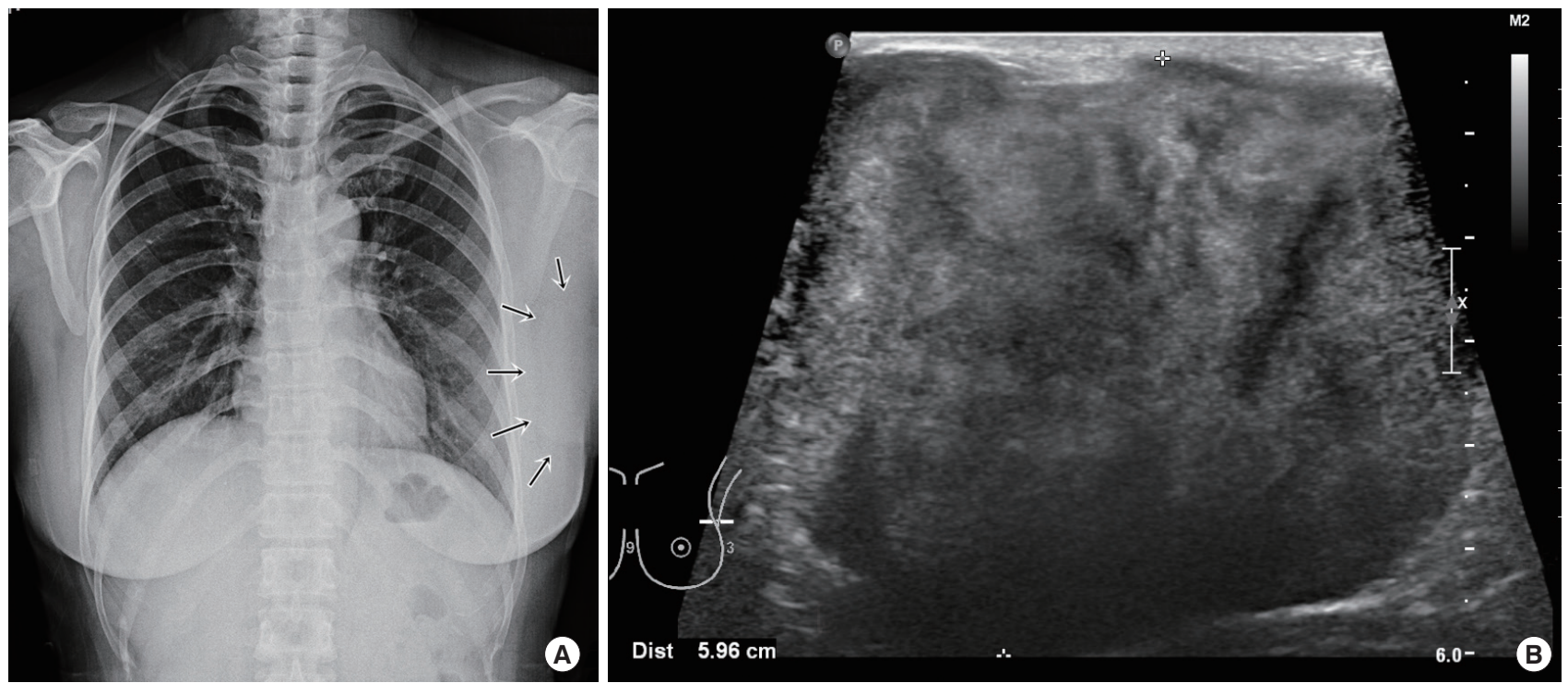

Figure 1. Chest posterior-anterior radiograph showing a mass shadow (arrows) in the left breast area (A) and ultrasonogram of left breast showing an approximately 10-cm sized homogenous enhancing solid mass (B).
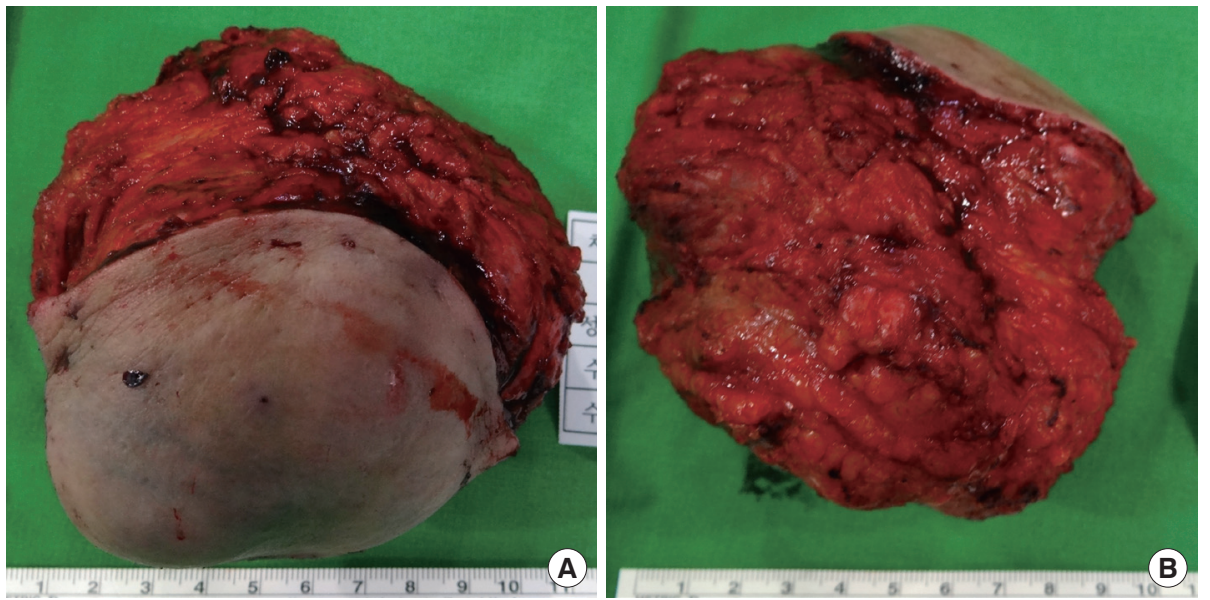

Figure 2. (A, B) Gross finding of initially resected left breast mass that measured $14.0 \times 8.0 \mathrm{~cm}$ in size.

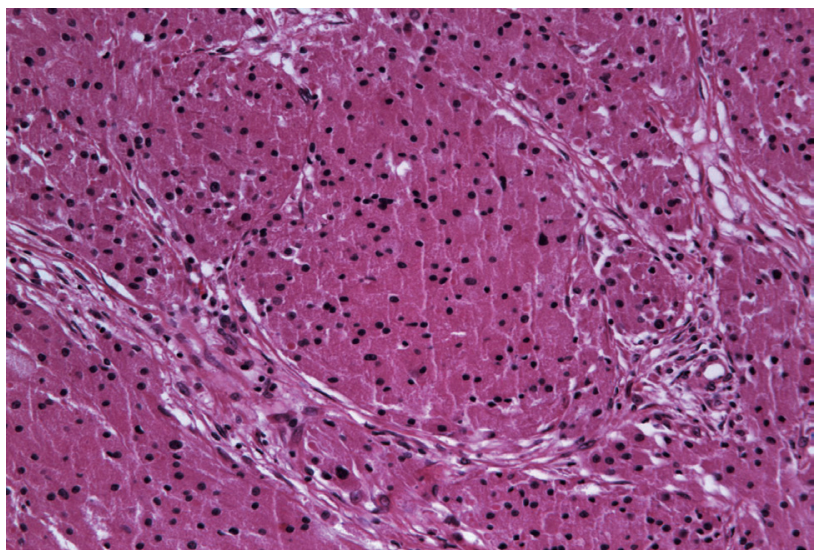

Figure 3. H\&E staining $(\times 200)$ of initially resected left breast mass showing eosinophilic granular cell proliferation with focal micronecrosis. after the first surgery. Pathologic results indicated benign GCTs with numerous aggregates of cells containing small round bland nuclei and abundant granular cytoplasm. Immunohistochemistry was strongly positive for S-100 (Figure 5).

\section{DISCUSSION}

GCT of the breast is relatively uncommon and has been reported to account for approximately 9\% of all GCTs [2]. First described in 1926 by Abrikossoff, GCT was initially presumed to be of myogenic origin, but recently, its strong immunoreactivity to $S-100$ protein revealed that it originates from Schwann cells $[5,6]$. In most cases, even in pa- 

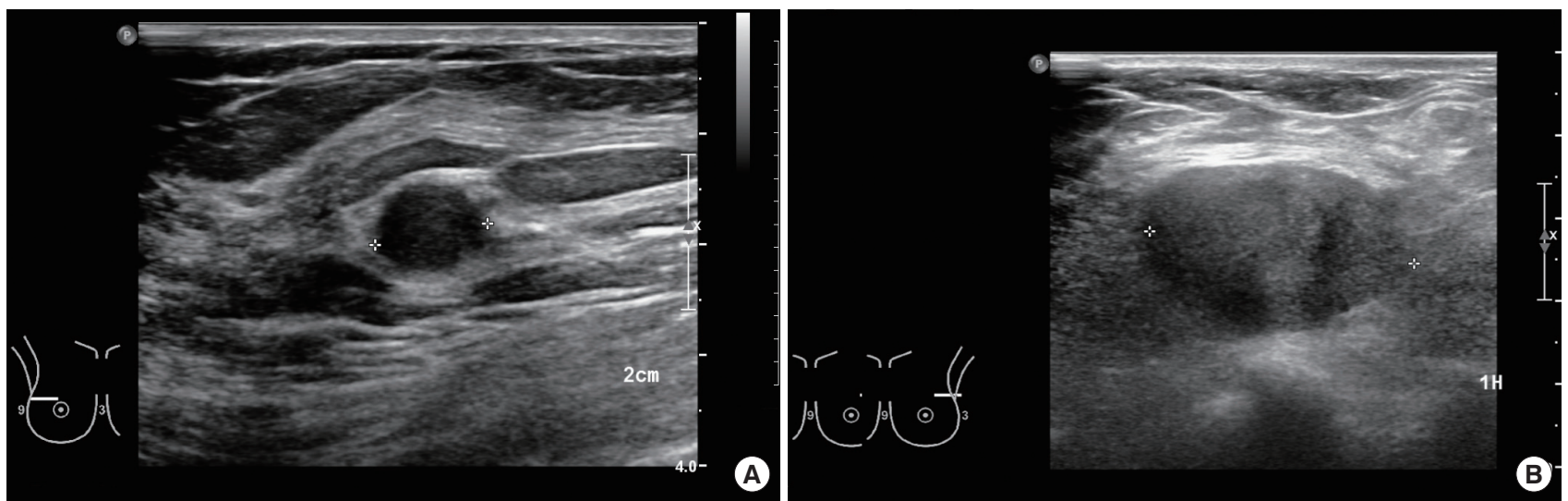

Figure 4. Ultrasonogram of the right breast showing a 1.2-cm sized mass with well-defined margins (A) and ultrasonogram of the left breast showing local recurrence of a $3-\mathrm{cm}$ sized mass in the previous operating site (B).
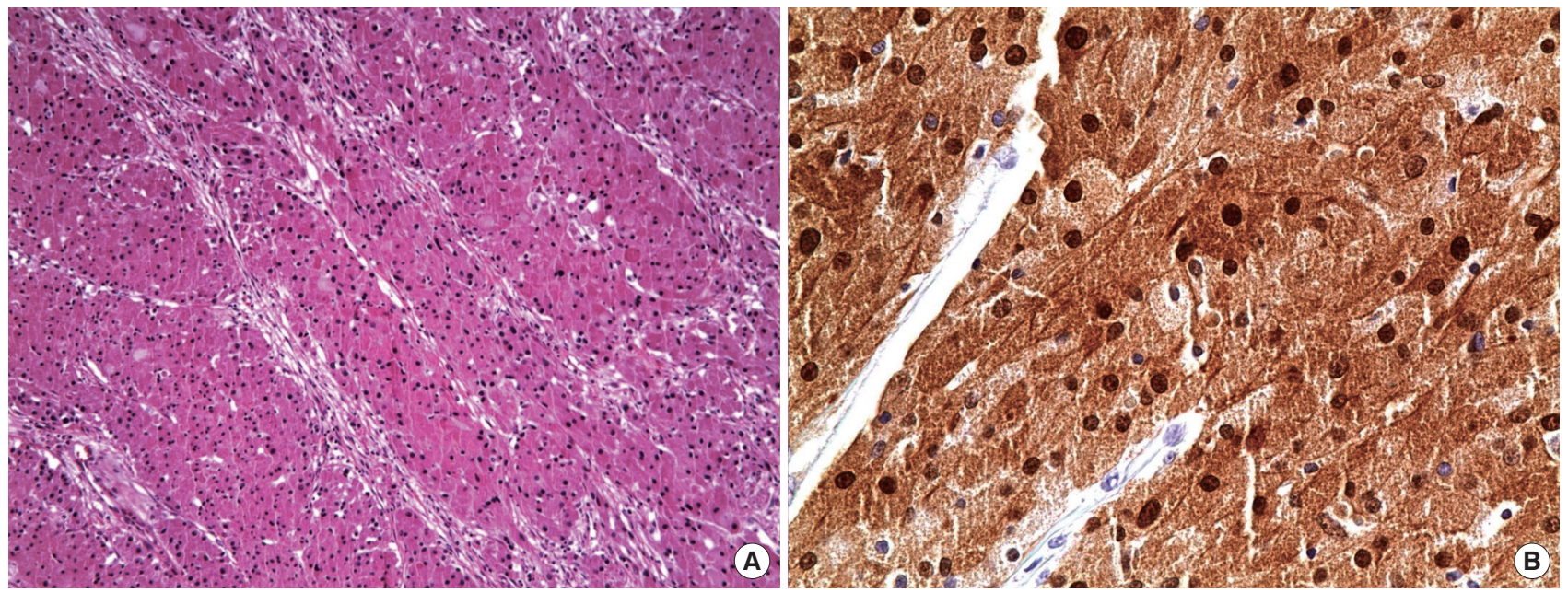

Figure 5. Hematoxylin and eosin staining $(\times 100)$ of specimens from the second surgery revealing numerous aggregates of cells containing small, round, bland nuclei, and abundant granular cytoplasm (A), and immunohistochemistry of right breast mass ( $\times 400)$ was strongly positive for S-100 (B).

tients with multiple and atypical GCTs, the tumor remains benign in nature, but in malignant cases, GCT is associated with a local recurrence rate of $32 \%$, a metastasis rate of $50 \%$, and mortality rate of $39 \%$ [6]. However, there are no specific findings to distinguish GCT from other types of breast lesions. Clinically, rapid growth, large tumor size $(>4 \mathrm{~cm})$, invasion into adjacent tissues, local recurrence, and an advanced age have been reported to be poor prognostic factors [7]. Nonspecific physical examination, mammographic, and ultrasound findings might not contribute to the diagnosis of GCT. In fact, only histopathologic confirmation after surgical excision provides a diagnosis of breast GCT. In the described case, the patient presented with a large left breast mass $(>10 \mathrm{~cm})$, and we were unable to determine whether it was malignant or benign based on ultrasonographic findings. For prompt diagnosis and treatment, we performed a wide excision rather than needle biopsy, and explained to the patient of the possibility of second operation based on the histopathologic results.

Fanburg-Smith et al. [6] suggest six histopathologic criteria for detecting malignant GCT. These features include high nuclear-to-cytoplasmic ratio, nuclear pleomorphism, necrosis, spindle-shaped tumor cells, vesicular nuclei with prominent nucleoli, and increased mitotic activity (>2 mitoses per 10 high-power fields at $200 \times$ magnification). Based on the above criteria, GCTs are classified as benign (satisfy 0 criteria or focal pleomorphism), atypical (satisfy 1-2 criteria), or malignant (satisfy more than 3 criteria) [6]. Furthermore, high Ki-67 expression was revealed in a majority of malignant cases [8]. In this case, only focal necrosis was consistent with malignant GCT that we classi- 
fied as benign. Despite histopathologic confirmation of the benign nature of the GCT, and the clear surgical margins achieved, the left side mass recurred and a new right side mass developed a year after the initial surgery. The recurrence rate of benign GCT is $2 \%$ to $8 \%$ with a clear resection margin, and this increases to $20 \%$ when resection margins are positive for tumor infiltration [6]. Our patient had some poor prognostic factors, such as large tumor size, local recurrence, and a new mass arising in the right breast. The treatment of GCT is clear and straightforward. Even for malignant GCT, only surgical resection is recommended. Chemotherapy and/or radiotherapy do not improve clinical course [9]. We also performed wide excision of GCTs in both breasts.

GCT of the breast is a rare neoplasm that rarely results in de novo development in the contralateral breast. It is difficult to differentiate benign and malignant lesions based on physical examination or imaging findings alone, and thus, initial diagnosis should further be confirmed by biopsy or complete surgical resection.

\section{CONFLICT OF INTEREST}

The authors declare that they have no competing interests.

\section{REFERENCES}

1. Radaelli F, Minoli G. Granular cell tumors of the gastrointestinal tract: questions and answers. Gastroenterol Hepatol (N Y) 2009;5:798-800.

2. Lack EE, Worsham GF, Callihan MD, Crawford BE, Klappenbach S, Rowden G, et al. Granular cell tumor: a clinicopathologic study of 110 patients. J Surg Oncol 1980;13:301-16.

3. Ilvan S, Ustündağ N, Calay Z, Bükey Y. Benign granular-cell tumour of the breast. Can J Surg 2005;48:155-6.

4. Aoyama K, Kamio T, Hirano A, Seshimo A, Kameoka S. Granular cell tumors: a report of six cases. World J Surg Oncol 2012;10:204.

5. Le BH, Boyer PJ, Lewis JE, Kapadia SB. Granular cell tumor: immunohistochemical assessment of inhibin-alpha, protein gene product 9.5, S100 protein, CD68, and Ki-67 proliferative index with clinical correlation. Arch Pathol Lab Med 2004;128:771-5.

6. Fanburg-Smith JC, Meis-Kindblom JM, Fante R, Kindblom LG. Malignant granular cell tumor of soft tissue: diagnostic criteria and clinicopathologic correlation. Am J Surg Pathol 1998;22:779-94.

7. Althausen AM, Kowalski DP, Ludwig ME, Curry SL, Greene JF. Granular cell tumors: a new clinically important histologic finding. Gynecol Oncol 2000;77:310-3.

8. Nasser H, Ahmed Y, Szpunar SM, Kowalski PJ. Malignant granular cell tumor: a look into the diagnostic criteria. Pathol Res Pract 2011;207:164-8.

9. Wang J, Zhu XZ, Zhang RY. Malignant granular cell tumor: a clinicopathologic analysis of 10 cases with review of literature. Zhonghua Bing Li Xue Za Zhi 2004;33:497-502. 